Research Article

\title{
Metabolomics of Hydrazine-Induced Hepatotoxicity in Rats for Discovering Potential Biomarkers
}

\author{
Zhuoling An $\mathbb{D}^{1},{ }^{1}$ Chao Li $\left(\mathbb{D},{ }^{2}\right.$ Yali Lv $\mathbb{D}^{1},{ }^{1}$ Pengfei Li ${ }^{D},{ }^{1}$ Cheng Wu, ${ }^{2}$ and Lihong Liu ${ }^{1}$ \\ ${ }^{1}$ Pharmacy Department of Beijing Chao-Yang Hospital Affiliated with Beijing Capital Medical University, Beijing, China \\ ${ }^{2}$ Pharmacy Department of the Second Artillery General Hospital of Chinese People's Liberation Army, Beijing, China \\ Correspondence should be addressed to Lihong Liu; hongllh@126.com
}

Received 15 August 2017; Accepted 20 November 2017; Published 10 April 2018

Academic Editor: Donald H. Chace

Copyright (C) 2018 Zhuoling An et al. This is an open access article distributed under the Creative Commons Attribution License, which permits unrestricted use, distribution, and reproduction in any medium, provided the original work is properly cited.

\begin{abstract}
Metabolic pathway disturbances associated with drug-induced liver injury remain unsatisfactorily characterized. Diagnostic biomarkers for hepatotoxicity have been used to minimize drug-induced liver injury and to increase the clinical safety. A metabolomics strategy using rapid-resolution liquid chromatography/tandem mass spectrometry (RRLC-MS/MS) analyses and multivariate statistics was implemented to identify potential biomarkers for hydrazine-induced hepatotoxicity. The global serum and urine metabolomics of 30 hydrazine-treated rats at 24 or $48 \mathrm{~h}$ postdosing and 24 healthy rats were characterized by a metabolomics approach. Multivariate statistical data analyses and receiver operating characteristic (ROC) curves were performed to identify the most significantly altered metabolites. The 16 most significant potential biomarkers were identified to be closely related to hydrazine-induced liver injury. The combination of these biomarkers had an area under the curve (AUC) $>0.85$, with $100 \%$ specificity and sensitivity, respectively. This high-quality classification group included amino acids and their derivatives, glutathione metabolites, vitamins, fatty acids, intermediates of pyrimidine metabolism, and lipids. Additionally, metabolomics pathway analyses confirmed that phenylalanine, tyrosine, and tryptophan biosynthesis as well as tyrosine metabolism had great interactions with hydrazine-induced liver injury in rats. These discriminating metabolites might be useful in understanding the pathogenesis mechanisms of liver injury and provide good prospects for drug-induced liver injury diagnosis clinically.
\end{abstract}

\section{Introduction}

Drug-induced liver injury is a life-threatening risk that is unpredictable and frequent during the clinical course of disease treatment [1-3]. The commonly used medicines as well as herbal products and vitamins may lead to liver injury [4-7]. The current methods for drug-induced liver injury diagnosis, such as blood biochemistry parameters including alanine aminotransferase (ALT), alkaline phosphatase (ALP), and aspartate aminotransferase (AST), are incapable of providing an accurate early diagnosis [8]. Hydrazine has been extensively applied as the classical animal model of liver injury and can also induce the alterations of endogenous metabolites including 2 -aminoadipate, $\beta$-alanine, 2 -oxoglutarate, and citrate [9-14]. However, to date, the reliability of biomarkers has not been verified and evaluated by multivariate data analysis methods or other methods of data analysis $[15,16]$. Therefore, it is still necessary to identify and validate reliable biomarkers by using integrated detection methods and analysis methods for hydrazine-induced hepatotoxicity. Metabolomics is reported to be a powerful quantitative approach for determining global metabolite changes in response to disease or medical treatment and is particularly suitable for discovering biomarkers in a complex system [17-21]. Various techniques and methods have been applied for identification of endogenous metabolites for metabolomics analyses, including liquid chromatography-mass spectrometry (LC-MS) in addition to gas chromatography-mass spectrometry (GC-MS) and ${ }^{1} \mathrm{H}$ nuclear magnetic resonance $\left({ }^{1} \mathrm{H}-\mathrm{NMR}\right)$ spectrometry [22-25]. The high sensitivity and potential for metabolite identification have made mass spectrometry (MS) the dominant approach for metabolomics studies [26, 27]. Previous research has shown that alterations of amino acids, glucose metabolism, lipid metabolism, and 
oxidative stress might be associated with hepatic fatty degeneration and glycogen accumulation, obtained mainly by using NMR- and GC-MS-based approaches [9-14]. These platforms or technologies have different strengths and weaknesses. Metabolomics studies currently lack a comprehensive metabolite identifying ability due to the lack of an electronic database. Multiple metabolomics platforms and technologies have allowed us to substantially enhance the level of metabolome coverage $[28,29]$. Up to now, potential biomarkers for drug-induced injury lack methodology validation by other omics or metabolic pathways as well as large clinical samples, and the predictive ability of the analytical platform is not fully understood. Potential biomarkers capable of providing an accurate early diagnosis for drug-induced liver injury still require further mining. Thus, metabolomics based on LCMS analyses may provide a comprehensive list of potential biomarkers for hydrazine-induced hepatotoxicity.

The present study utilized a nontargeted metabolomics approach by rapid-resolution liquid chromatography-mass spectrometry (RRLC-MS) combined with multivariate statistical analyses to investigate the metabolic profile changes between healthy and hydrazine-administered rats. Metabolic disturbances and changes in response to pathological conditions were further evaluated by multivariate statistics to identify potential diagnostic biomarkers. The reliabilities of the identified discriminated metabolites were further validated by receiver operating characteristic (ROC) analyses.

\section{Experimental Procedures}

2.1. Chemicals. Hydrazine hydrate was from Acros Organics (Morris Plains, NJ, USA). Acetonitrile and formic acid (HPLC-grade) were from Merck (Darmstadt, Germany). Metabolite standards with 99\% purity (creatine, L-carnitine, tryptophan, tyrosine, kynurenic acid, adenosine, tanshinone IIA, peoniflorin, isosorbide 5-mononitrate, digitoxin, and tramadol) were from Sigma-Aldrich. All other standards used were of analytical or higher grade.

2.2. Animal Study Design and Sample Collection. Male Wistar rats ( $n=60,6-7$ weeks old) were from Charles River China Inc. (Vital River Laboratories, China). The experiments were carried out under the approval by the Animal Ethics Committee of Beijing Chao-Yang Hospital affiliated with Beijing Capital Medical University. Rats were randomly divided into four groups: two control groups (12 rats/group) and two hydrazine-treated groups (18 rats/group). One control group and one hydrazine-treated group were allocated for sampling at $24 \mathrm{~h}$ postdosing, while the remaining two groups were for sampling at $48 \mathrm{~h}$ postdosing. The hydrazine-treated groups were orally administrated with a single dose of hydrazine $(150 \mathrm{mg} / \mathrm{kg})$, at which hydrazine could induce an obvious histopathological effect and hepatocellular lipid accumulation [10-14].

Urine samples from metabolic cages were collected for $8 \mathrm{~h}$ from 16 to $24 \mathrm{~h}$ and 40 to $48 \mathrm{~h}$ postdosing and then centrifuged at $5000 \times \mathrm{g}$ for $5 \mathrm{~min}$ at $4^{\circ} \mathrm{C}$; the supernatant was used for creatinine concentration measurement [14].
The orbital blood was taken from rats at 24 and $48 \mathrm{~h}$ postdosing. Serum samples collected by centrifugation at $5000 \times \mathrm{g}$, and $4^{\circ} \mathrm{C}$ for $5 \mathrm{~min}$ was used for determining ALT, ALP, and AST concentrations. Blood biochemistry analyses were measured by a clinical biochemistry analyzer (AU400, Olympus, Japan) at De Yi Biotechnology Co. Ltd.

After blood collection, the rat livers were collected and fixed in $4 \%$ formaldehyde. The fixed rat liver samples were trimmed, embedded in paraffin wax, sectioned, and then stained with hematoxylin and eosin (H\&E) for histopathological examination.

2.3. Sample Preparation. An aliquot of $50 \mu \mathrm{L}$ of urine supernatant was further diluted to $200 \mu \mathrm{L}$ with water and then filtered through a syringe filter $(0.2 \mu \mathrm{m}, 96$-well Captiva sample prep solutions, Agilent Technologies, USA) before LC-MS analysis. A $100 \mu \mathrm{L}$ aliquot of serum was added to $300 \mu \mathrm{L}$ of cold acetonitrile, and the mixture was vortexed for $4 \mathrm{~min}$ and centrifuged at $12,000 \times \mathrm{g}$ for $10 \mathrm{~min}$. A total of $200 \mu \mathrm{L}$ of the supernatant was centrifuged (SAVANT SPD121 P SpeedVac, Thermo Scientific, USA) for $60 \mathrm{~min}$ or $90 \mathrm{~min}$ at $4^{\circ} \mathrm{C}$. Dry residues were reconstituted in $200 \mu \mathrm{L}$ of water containing $2 \%$ acetonitrile and were vortexed for $5 \mathrm{~min}$. The supernatant was removed and filtered through a syringe filter for RRLC-MS analysis.

2.4. RRLC-MS Analysis. Chromatographic separation was performed on an ACQUITY UPLC HSS $\mathrm{T}_{3}$ column $(1.8 \mu \mathrm{m}$, $10 \mathrm{~cm} \times 2.1 \mathrm{~mm}$, Waters, Ireland) with an Agilent $1200 \mathrm{RRLC}$ system (Agilent Technologies, Aldbronn, Germany). The column temperature was kept at $50^{\circ} \mathrm{C}$, and the injection volume was $5 \mu \mathrm{L}$. The analysis gradient conditions for both serum and urine samples were the same, as follows: 0-5 min, linear gradient of 2-10\% B; 5-17 min, linear gradient of $10-40 \% \mathrm{~B} ; 17-20 \mathrm{~min}, 40-100 \% \mathrm{~B}$; and $20-30 \mathrm{~min}$, $100 \% \mathrm{~B}$. The program was followed by a return to the starting conditions, which were maintained for $8 \mathrm{~min}$ to equilibrate the column. Mobile phase A was $0.1 \%$ formic acid in water, and mobile phase $\mathrm{B}$ was acetonitrile. The flow rate was $250 \mu \mathrm{L} / \mathrm{min}$.

MS spectra were acquired on a Q-TOF LC-MS system (AB/MDS Sciex, Foster City, CA) with an electrospray ion (ESI) source in positive-ion mode. The ion source voltage was $5.5 \mathrm{kV}$, and the declustering potential was $50 \mathrm{~V}$. The collision energy (CE) was set to $30 / 10 \mathrm{eV}$, and the vaporizer temperature was $450^{\circ} \mathrm{C}$. The MS was operated with gas conditions of $30 \mathrm{psi}$ for the curtain gas, 5 psi for the collision gas, 70 psi for the nebulizer gas (GS1), and 60 psi for the drying gas (GS2). Nitrogen gas was used as both the nebulizing and drying gas. The mass-to-charge $(\mathrm{m} / \mathrm{z})$ scan range was from 65 to 1000. Data processing and acquisition were conducted on Analyst QS 2.0 software (QSTAR Elite, AB/MDS Sciex).

MS/MS spectra were obtained with informationdependent acquisition mode. MS signals were first collected by one time-of-flight (TOF) survey scan and followed by two product-ion scans for parent ions with the highest intensity. For each survey scan, three MS/MS experiments were triggered. Compounds with identical $\mathrm{m} / \mathrm{z}$ ratios were 
excluded automatically. Metabolites of interest were added to the MS/MS table list. MS/MS data were auto-normalized by background ions (phthalates: $m / z 391.2843$ and 149.0233).

2.5. Assessment for Data Quality. To obtain high-quality and reliable data to reflect the endogenous metabolite changes, analytical and technical errors should be decreased to minimize any influence on multivariate data analysis. In the current study, a test standard mixture, including adenosine, tanshinone IIA, peoniflorin, isosorbide 5-mononitrate, digitoxin, and tramadol, was added into the sample batch to monitor the system stability. Quality control (QC) samples prepared by pooling equal volumes of urine or serum from each healthy and liver-injured rat were treated as the real samples to monitor system reproducibility and stability [27].

2.6. Data Processing. RRLC-MS raw data were converted to the $\mathrm{m} / z$ format with the threshold set at $1 \%$ using wiff to $\mathrm{m} / \mathrm{z}$ data translator software (version 1.0.0.4, AB/MDS Sciex). Using open-source software MZmine 2 beta (version 2.9.1), peak finding, alignment, filtering, and scaling were carried out. All parameters were optimized in a stepwise manner until aligned peaks agreed with those from manual inspection [30, 31]. Detailed preprocessing parameters are listed in S1 Table [22].

The preprocessing data from urine samples were normalized by the creatinine level. The obtained data were then subjected to SIMCA-P 13.0 software (Umetrics AB, Umeå, Sweden) for multivariate data analysis. In order to reduce model noise and artifacts, centered- and pareto-scaling were used prior to multivariate statistical analysis. To test the model validity against overfitting and to validate the biomarkers statistically, $Q^{2}$, the cross-validation parameter, was calculated using partial least squares discriminant analysis (PLS-DA) by a random permutation test of 100 permutations. Discriminating variables were chosen based on jack-knife confidence intervals, S-plot, variable importance on projection (VIP) values, and raw data plots using the orthogonal partial least squares discriminant analysis (OPLS-DA) model. The OPLS-DA model quality was evaluated by the relevant $R^{2}$ and $Q^{2}$ values as well as the intercepts of $R^{2}$ and $Q^{2}$. The OPLS-DA models were considered to be valid only if $0<R^{2}(Y)-Q^{2}(Y)<0.3, Q^{2}(Y)>0.5$, intercepts of $R^{2}<0.4$, and intercepts of $Q^{2}<0.05$ [32]. The independent $t$-test (2007 version of Microsoft Office Excel) was used to check whether potential biomarkers derived from OPLSDA modeling were statistically significant $(p<0.05)$.

Metabolites were identified via searching free databases including HMDB, PubChem, METLIN, and KEGG, using accurate molecular weights [33]. High-resolution mass spectra (HR-MS/MS) were applied for further identification. Based on the retention time comparison, authentic standards were also used. Each identified biomarker was evaluated for its discriminatory power and predictive ability using the area under the receiver operating characteristic curve [34]. Metabolic pathway analysis for potential biomarkers was carried out by powerful pathway enrichment analysis and pathway topology analysis to identify the most relevant pathways through MetaboAnalyst 3.0 (http://www.metaboanalyst.ca).
TABLE 1: Alterations of blood biochemistry parameters in hydrazine-treated rats.

\begin{tabular}{ccccc}
\hline Time & Dose & ALT (U/L) & ALP (U/L) & AST (U/L) \\
\hline \multirow{2}{*}{$24 \mathrm{~h}$} & $0 \mathrm{mg} / \mathrm{kg}$ & $48.4 \pm 14.8$ & $199.5 \pm 21.4$ & $150.7 \pm 34.9$ \\
& $150 \mathrm{mg} / \mathrm{kg}$ & $39.8 \pm 10.2$ & $180.1 \pm 24.1^{*}$ & $109.8 \pm 55.5^{*}$ \\
\hline \multirow{2}{*}{$48 \mathrm{~h}$} & $0 \mathrm{mg} / \mathrm{kg}$ & $47.3 \pm 19.4$ & $193.1 \pm 41.0$ & $131.2 \pm 18.0$ \\
& $150 \mathrm{mg} / \mathrm{kg}$ & $31.3 \pm 15.5^{\dagger}$ & $201.1 \pm 27.5$ & $86.6 \pm 45.6^{\dagger \dagger}$ \\
\hline
\end{tabular}

ALT: alanine aminotransferase; ALP: alkaline phosphatase; AST: aspartate aminotransferase. *Significantly different from the $0 \mathrm{mg} / \mathrm{kg}$ group $(24 \mathrm{~h})$ $(p<0.05)$. $†$ Significantly different from the $0 \mathrm{mg} / \mathrm{kg}$ group $(48 \mathrm{~h})(p<0.05)$. $\dagger \dagger$ Significantly different from the $0 \mathrm{mg} / \mathrm{kg}$ group $(48 \mathrm{~h})(p<0.01)$.

The most relevant pathways involved were confirmed only if the pathway impact was greater than 0.1 and the node size and color were graphically larger and darker.

\section{Results and Discussion}

3.1. Toxicological Examination. Hydrazine-induced hepatotoxicity in rats was confirmed by evaluation of blood biochemistry and liver tissue histology. Alternations of the blood biochemical parameters between healthy and hydrazine-treated rats are listed in Table 1. AST decreased significantly in the hydrazine-treated rats at both 24 and $48 \mathrm{~h}$ postdosing, perhaps owing to hydrazine or its metabolites causing sequestration of the aminotransferase cofactor pyridoxal 5-phosphate, resulting in inhibitory effects on aminotransferase activities [9-14, 35]. However, ALT and ALP did not significantly differ between hydrazine-treated groups at 24 and $48 \mathrm{~h}$ postdosing and their controls, respectively, indicating hepatic injuries might be accompanied with little changes of ALT and ALP for self-regulation and recovery. The results also suggested that the activities of hepatotoxicity enzymes might not be sensitive enough to predict hydrazine-induced liver injury.

The histopathological photographs are shown in Figure 1. Figures 1(a) and 1(b) show normal hepatocytes surrounding the central vein in control rats, and Figures 1(c)-1(f) show the cytoplasmic vacuoles induced by fatty degenerations in the midzonal area of hepatocytes and single-cell necrosis of hepatocytes in hydrazine-treated rats. Additionally, isolated extracellular and eosinophilic bodies were distributed in some necrotic cells of hydrazine-treated rats. These findings indicate that hydrazine-treated rats at 24 or $48 \mathrm{~h}$ postdosing had suffered from serious liver injury.

3.2. Assessment of Data Quality. To obtain high-quality and reliable data to reflect the endogenous metabolite changes, analytical and technical errors should be decreased to minimize any influence on multivariate data analysis. In the current study, a test standard mixture including adenosine, tanshinone IIA, peoniflorin, isosorbide 5-mononitrate, digitoxin, and tramadol was added into the sample batch to monitor the system stability. As demonstrated in S1 Fig, the maximum deviation in retention time for all standards in the test standard mixture was $0.06 \mathrm{~min}$, the average variation of retention time was $<0.82 \%$ (RSD), 


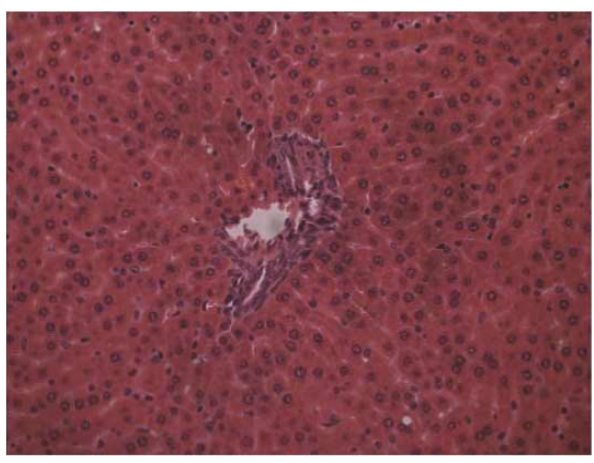

(a)

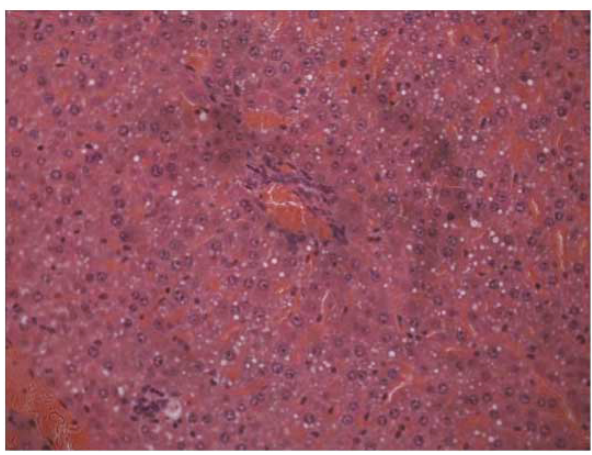

(c)

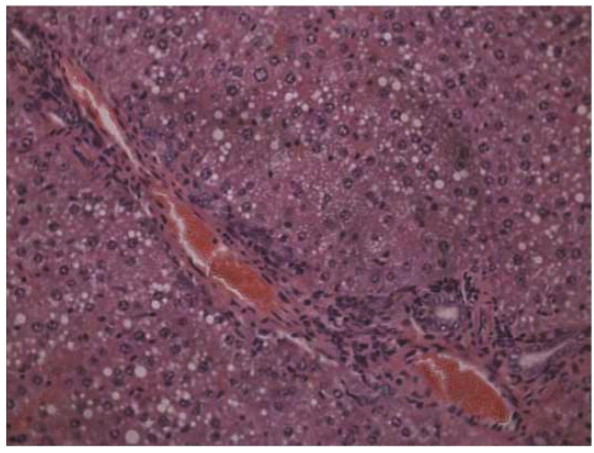

(e)

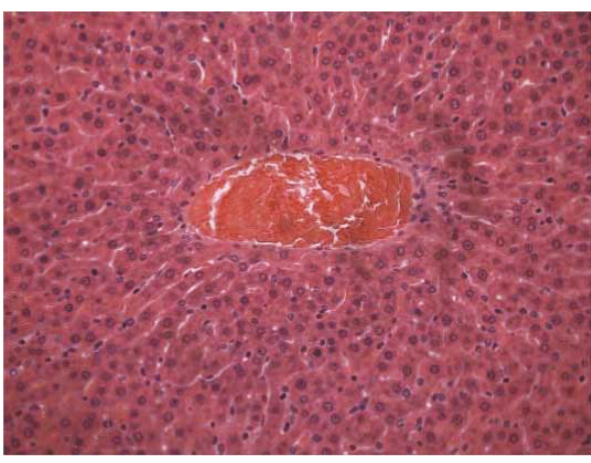

(b)

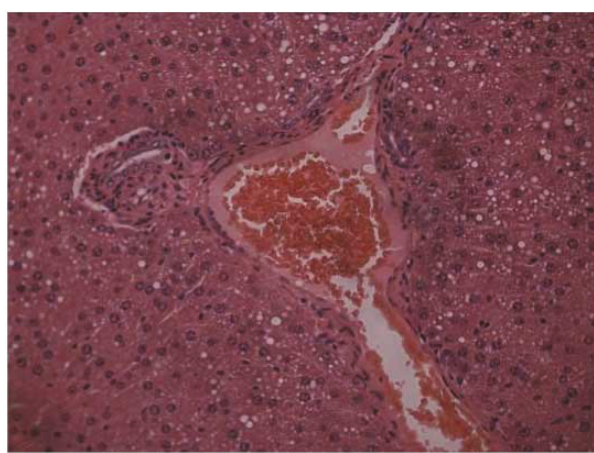

(d)

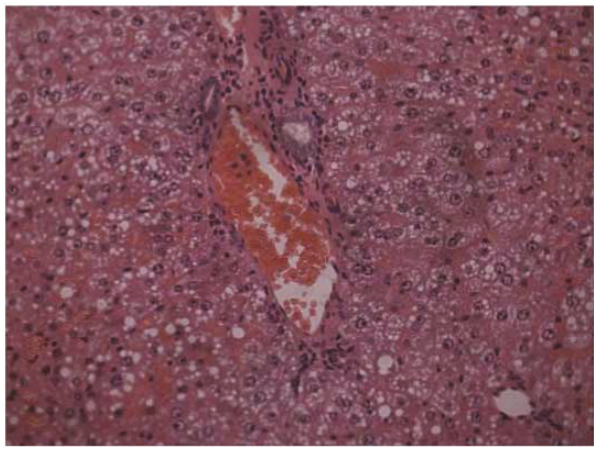

(f)

FIgURE 1: Liver histopathology at $24 \mathrm{~h}$ and $48 \mathrm{~h}$ postdosing for control rats and liver-injured rats, which were induced by hydrazine. No abnormalities were detected in the controls $(0 \mathrm{mg} / \mathrm{kg}$ ) at the $24 \mathrm{~h}$ and $48 \mathrm{~h}$ time points ( $\mathrm{a}$ and b). Fatty degeneration and single-cell necrosis appeared obviously in the midzonal areas of the hydrazine-treated groups $(150 \mathrm{mg} / \mathrm{kg})$ at $24 \mathrm{~h} \mathrm{(c} \mathrm{and} \mathrm{d)} \mathrm{and} 48 \mathrm{~h}(\mathrm{e}$ and f) postdosing. Histological sections were stained with $H \& E(\times 400)$.

and the average variation of extracted ion areas was $<14.30 \%$ (RSD) (S2 Table). Multivariate analysis was performed after creatinine normalization and unit variance scaling. Using principal component analysis (PCA), the peak area deviation of serum or urine QC plots was less than 2-fold of the SD (S2 Fig). These results indicate that differences between test samples were more likely to reflect varied metabolic profiles than technical errors or analytical variation.

3.3. Multivariate Statistical Analysis of Control and LiverInjured Rats. Using MZmine software, the RRLC-MS data of urine and serum samples were analyzed by peak detection and integration, leading to the identification of $\mathrm{m} / z 647$ and $m / z 604$ peaks for urine and serum samples between retention times of 1 to $25 \mathrm{~min}$, respectively.

First, PCA was applied to integrate and coanalyze all observations from serum and urine samples to investigate metabolomics changes. The control groups and hydrazinetreated groups at both $24 \mathrm{~h}$ and $48 \mathrm{~h}$ postdosing were not well distinguished in terms of PCA score plots, and the $48 \mathrm{~h}$ postdosing group did not show a significant difference compared with the $24 \mathrm{~h}$ postdosing group, especially for the urine samples. OPLS-DA was applied to the control groups and hydrazine-treated groups at both $24 \mathrm{~h}$ and $48 \mathrm{~h}$ postdosing. The separation of the three groups was good (Figure 2). Furthermore, OPLS-DA was applied as a stoichiometric analysis method to explore 


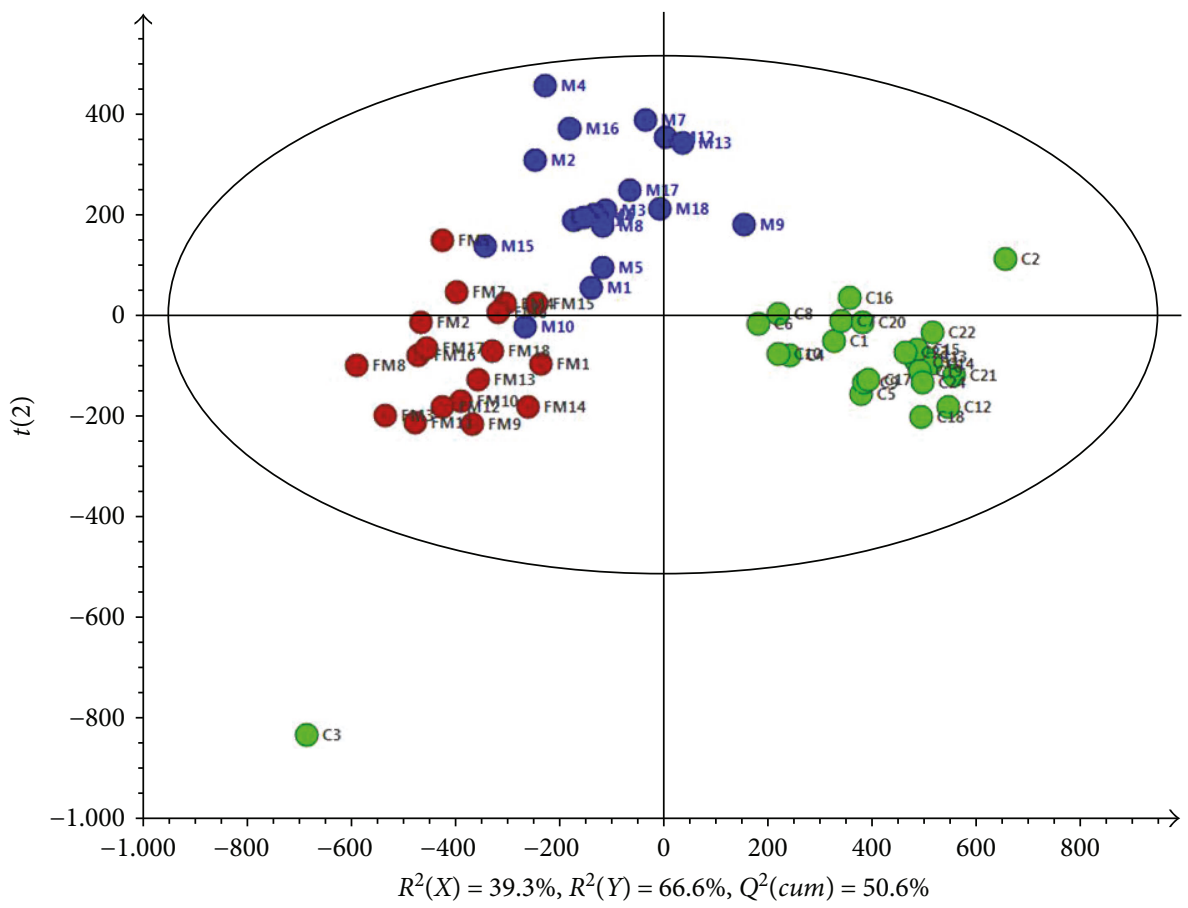

(a)

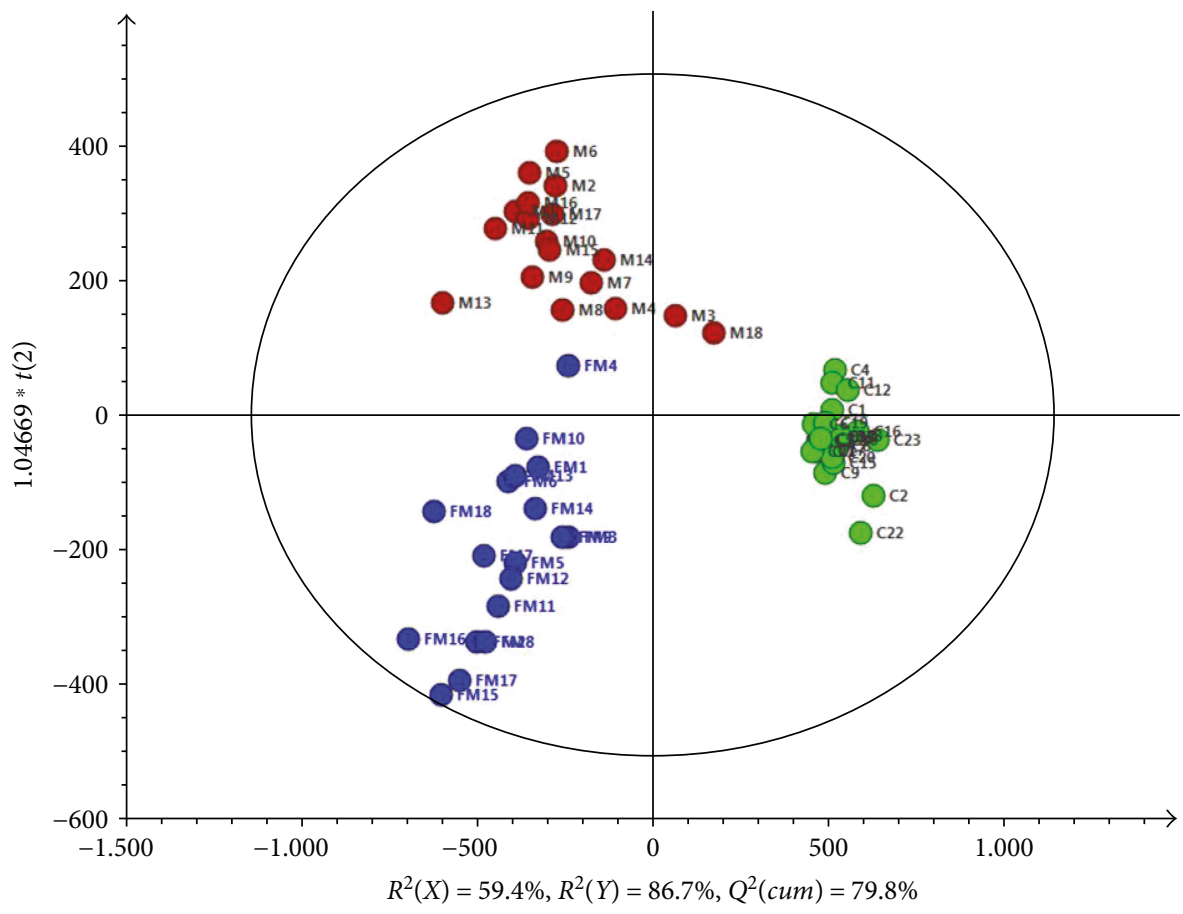

(b)

FIGURE 2: OPLS-DA score plots of (a) serum and (b) urine samples derived from the RRLC-(+) ESIMS data. (The symbols are as follows: green circle $=\mathrm{C}$ healthy rats; blue circle $=\mathrm{M}$ liver-injured rats induced by hydrazine at $24 \mathrm{~h}$ postdosing; and brown circle $=\mathrm{FM}$ liverinjured rats induced by hydrazine at $48 \mathrm{~h}$ postdosing.)

differences between healthy and liver-injured rats. Score plots for the OPLS-DA models showed a clear separation between healthy and liver-injured rats (Figure 3). For serum data analyses, one predictive component $\left(t_{\mathrm{p}}\right)$ and two orthogonal $\left(t_{\mathrm{o}}\right)$ components $(1+2)$ with cross-validated predictive abilities $Q^{2}$ (cum) of $85.9 \%$ were derived.
Additionally, $38.9 \%$ of the variance in $X\left[R^{2}(X)\right]$ was applied to account for $92 \%$ of the $Y\left[R^{2}(Y)\right]$ variance (Figure 3(a)). For urine data analyses, $R^{2}(X)=41.1 \%$, $R^{2}(Y)=90.5 \%$, and $Q^{2}(\mathrm{cum})=86.8 \%$ across one predictive component $\left(t_{\mathrm{p}}\right)$ and two orthogonal $\left(t_{\mathrm{o}}\right)$ components are shown in Figure 3(b). 


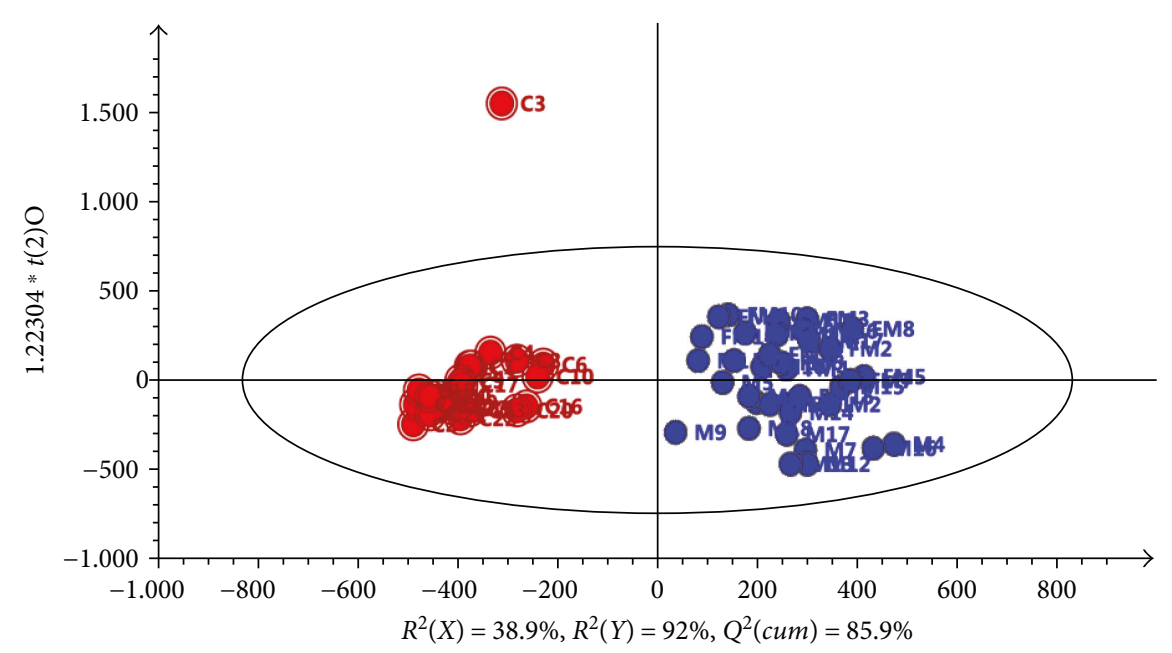

(a)

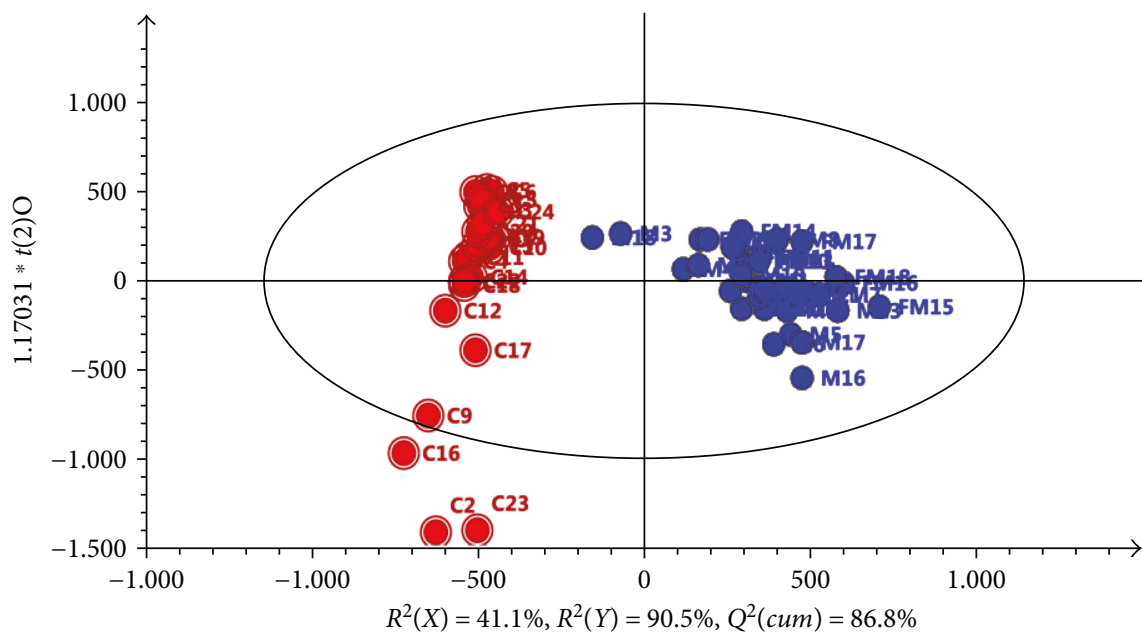

(b)

FIgURE 3: OPLS-DA score plots derived from the RRLC-(+) ESIMS data from (a) serum and (b) urine samples. (The symbols are as follows: red circle $=\mathrm{C}$ healthy rats and blue circle $=\mathrm{M}$ and $\mathrm{FM}$ liver-injured rats induced by hydrazine at $24 \mathrm{~h}$ and $48 \mathrm{~h}$ postdosing.)

To avoid overfitting, a seven-round validation across three components by excluding $1 / 7$ th of the samples in each round was applied. Validation by 100 random permutation tests resulted in intercepts of $R^{2}=0.267$ and $Q^{2}=-0.235$ for the serum data (S3 Fig A and B). Similarly, the validated intercepts of $R^{2}=0.213$ and $Q^{2}=-0.242$ were calculated for the urine data (S3 Fig C and D). These results demonstrated that the OPLS-DA models based on the RRLC-ESI-MS data showed good predictability for both the serum and urine samples.

3.4. Screening of Potential Diagnostic Biomarker Candidates. S-plot was applied to screen the discriminative variables. Metabolites with a high correlation were selected preferentially [36]. Variables with a VIP $>1.0$ were marked as variables of interest [37]. Raw data plots and jack knife-based confidence interval analyses were subsequently employed to remove variables with low reliability. In parallel, the independent $t$-test was applied to evaluate the significance of the concentration difference for identified variables $(p<0.001)$. Redundant variables obtained from the same metabolite were excluded by partial correlation coefficients. Consequently, 27 and 53 metabolites detected in serum and urine, respectively, were deemed to be biomarker candidates reflecting metabolic differences. To further identify serumor urine-specific biomarker(s), the first screened potential biomarkers were evaluated by a self-compiled Microsoft Visual Basic program [22]. The results showed that only five discriminatory metabolites were identified in both serum and urine. In total, 22 and 48 metabolites were considered as unique biomarkers for serum and urine, respectively (S3 Table).

3.5. Identification of Potential Biomarkers by MS/MS Fragmentation Patterns. A molecular formula could be determined according to the accurate mass weights, mass defect considerations, assignments, MS/MS fragmentation patterns, and relative intensities of the isotope peaks obtained from the 


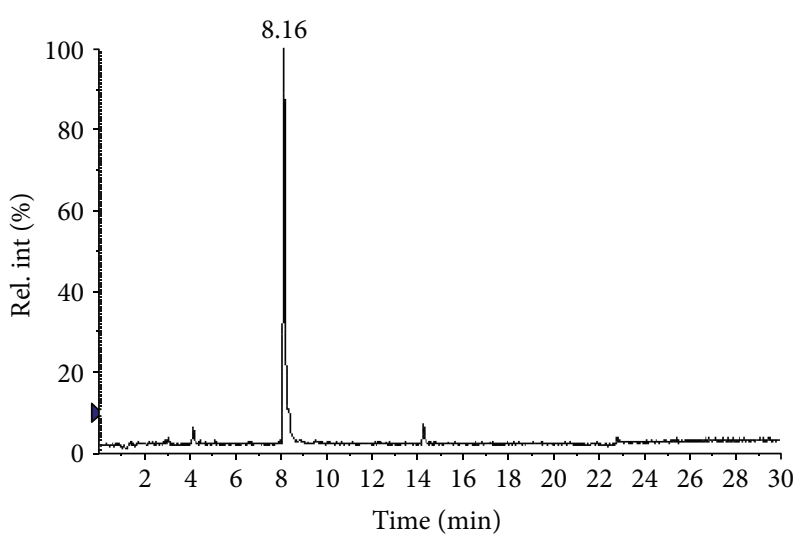

(a)

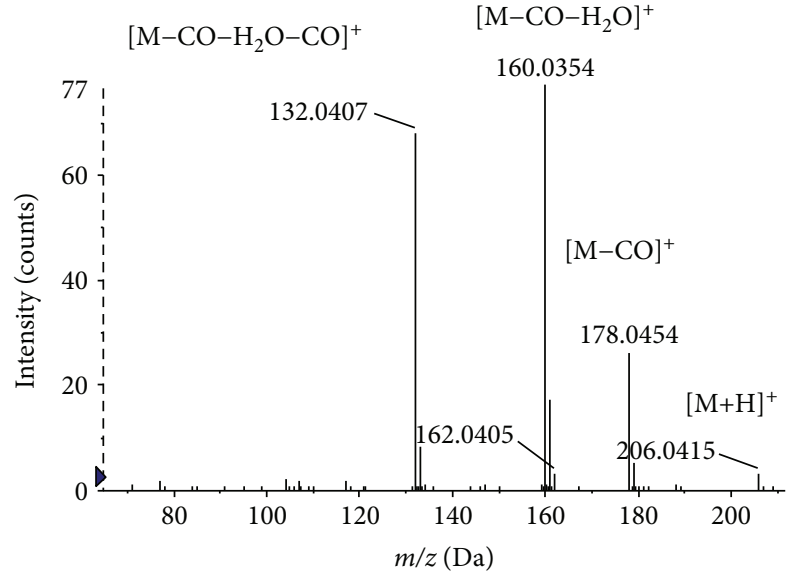

(b)<smiles>O=C(O)c1cc(O)c2cccc(O)c2[nH+]1</smiles>

$[\mathrm{M}+\mathrm{H}]^{+} m / z 206$<smiles>O=Cc1cccc2c(O)cc(O)[nH+]c12</smiles>

$[\mathrm{M}+\mathrm{H}]^{+} m / z 178$<smiles></smiles>

$[\mathrm{M}+\mathrm{H}]^{+} m / z 132$

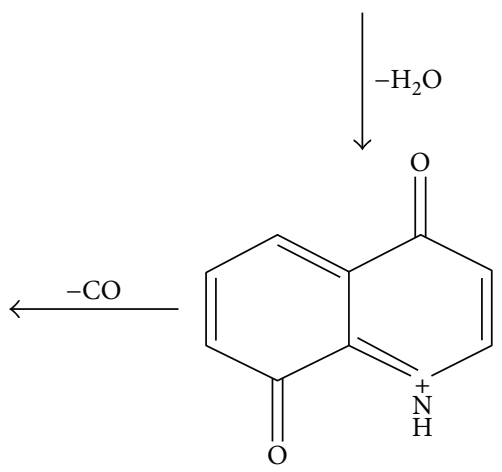

$[\mathrm{M}+\mathrm{H}]^{+} m / z 160$

(c)

Figure 4: (a) Extracted ion chromatogram (XIC) of $m / z 206.0415$ in positive-ion mode of LC-MS analysis for urine samples. (b and c) The identification of the metabolite xanthurenic acid by means of Q-TOF MS/MS in positive-ion mode; the positive product ion spectrum of $m / z$ 206.0415 at $8.16 \mathrm{~min}$ (b) and its postulated main fragmentation pathway (c).

HR-MS/MS [22, 31, 33]. Here, the identification processes were briefly demonstrated by taking $m / z 206.0415$ as an example (Figure 4). Such steps enabled the characterization of 19 potential biomarkers in the serum and urine. Their HR-MS/MS fragments and relative abundances are listed in Table 2. Five potential screened biomarkers (creatine, L-carnitine, tryptophan, tyrosine, and kynurenic acid) were further confirmed by the retention time comparison with the standard.

3.6. Characterization of Potential Diagnostic Biomarkers. ROC analyses were performed to reconfirm these putative biomarkers for their discriminatory power. Nineteen identified potential biomarkers ( 8 for serum, 12 for urine, and 1 for both serum and urine) were classified into two categories, 8 were upregulated, and 11 were downregulated in liverinjured rats (Table 2). The seven upregulated metabolites had AUC values of 0.667 to 1 (S4 Fig A). Only tyrosine showed a relatively low diagnostic significance $(A U C<0.7)$ $[31,34]$. The eleven downregulated metabolites provided AUC values of 0.838 to 1 (S4 Fig B). Heat maps were also obtained to evaluate the discriminative power of potential biomarkers, and their AUC values are ranked in Figure 5(a). The peak area changes between the control 
TABLE 2: Identified potential biomarkers related to perturbations of hydrazine-induced liver injury.

\begin{tabular}{|c|c|c|c|c|c|c|c|}
\hline $\begin{array}{l}\text { Biological } \\
\text { sample }\end{array}$ & $\begin{array}{l}\mathrm{RT} \\
(\mathrm{min})\end{array}$ & $m / z$ & $\begin{array}{l}\text { Elemental } \\
\text { composition }\end{array}$ & $\begin{array}{c}\text { Metabolite } \\
\text { identification }\end{array}$ & MS/MS fragments ${ }^{c}$ & $\begin{array}{c}\mathrm{VIP}^{d} \\
\text { (trend) }\end{array}$ & $p$ value $^{e}$ \\
\hline Serum & 2.19 & 130.0512 & $\mathrm{C}_{5} \mathrm{H}_{8} \mathrm{NO}_{3}^{+}$ & Pyroglutamic acid ${ }^{b}$ & 84.0449 & $1.03 \uparrow$ & $2.33 E-09$ \\
\hline Serum & 1.22 & 132.0770 & $\mathrm{C}_{4} \mathrm{H}_{10} \mathrm{~N}_{3} \mathrm{O}_{2}^{+}$ & Creatine $\mathrm{e}^{a, b}$ & $90.0555,87.0791,72.0565$ & $4.25 \uparrow$ & $9.83 E-11$ \\
\hline Serum & 1.26 & 144.1029 & $\mathrm{C}_{7} \mathrm{H}_{14} \mathrm{NO}_{2}^{+}$ & Prolinebetaine $^{b}$ & $102.0556,84.0812$ & $1.36 \downarrow$ & $3.44 E-14$ \\
\hline Serum & 1.16 & 162.1129 & $\mathrm{C}_{7} \mathrm{H}_{16} \mathrm{NO}_{3}^{+}$ & L-Carnitine $e^{a, b}$ & $103.0377,85.0277$ & $1.45 \uparrow$ & $3.15 E-13$ \\
\hline Serum & 1.14 & 198.0861 & $\mathrm{C}_{8} \mathrm{H}_{12} \mathrm{~N}_{3} \mathrm{O}_{3}^{+}$ & $N$-Acetylhistidine ${ }^{b}$ & $153.0890,138.0548$ & $1.62 \uparrow$ & $1.86 E-20$ \\
\hline Serum & 1.80 & 204.1243 & $\mathrm{C}_{9} \mathrm{H}_{18} \mathrm{NO}_{4}^{+}$ & L-Acetylcarnitine ${ }^{b}$ & $144.1032,85.0870$ & $1.25 \downarrow$ & $1.28 E-06$ \\
\hline Serum & 5.20 & 205.0990 & $\mathrm{C}_{11} \mathrm{H}_{13} \mathrm{~N}_{2} \mathrm{O}_{2}^{+}$ & Tryptophan $^{a, b}$ & $188.0704,146.0612,118.0673$ & $2.30 \uparrow$ & $1.21 E-09$ \\
\hline Serum/urine & 2.62 & 182.0803 & $\mathrm{C}_{9} \mathrm{H}_{12} \mathrm{NO}_{3}^{+}$ & Tyrosine $^{a, b}$ & $\begin{array}{l}165.0548,136.0760 \\
123.0449,119.0490\end{array}$ & $5.38 / 2.28 \uparrow$ & $\begin{array}{r}1.77 E-06 / \\
9.94 E-04\end{array}$ \\
\hline Urine & 1.51 & 127.0490 & $\mathrm{C}_{5} \mathrm{H}_{7} \mathrm{~N}_{2} \mathrm{O}_{2}^{+}$ & Thymine $^{b}$ & 84.0444 & $1.11 \downarrow$ & $5.05 E-11$ \\
\hline Urine & 1.69 & 130.0857 & $\mathrm{C}_{6} \mathrm{H}_{12} \mathrm{NO}_{2}^{+}$ & Pipecolic acid $b$ & 84.0808 & $2.28 \downarrow$ & $9.68 E-06$ \\
\hline Urine & 1.21 & 138.0534 & $\mathrm{C}_{7} \mathrm{H}_{8} \mathrm{NO}_{2}^{+}$ & Trigonelline $e^{b}$ & $94.0650,78.0332,67.0407$ & $1.96 \downarrow$ & $3.43 E-12$ \\
\hline Urine & 4.02 & 144.0660 & $\mathrm{C}_{6} \mathrm{H}_{11} \mathrm{NO}_{3}^{+}$ & Vinylacetylglycine $e^{b}$ & $99.0680,98.0568,86.0600$ & $7.35 \uparrow$ & $1.55 E-05$ \\
\hline Urine & 1.70 & 146.0913 & $\mathrm{C}_{5} \mathrm{H}_{12} \mathrm{~N}_{3} \mathrm{O}_{2}^{+}$ & 4-Guanidinobutanoic acid ${ }^{b}$ & $\begin{array}{c}111.0566,87.0440 \\
86.0584,69.0326\end{array}$ & $2.42 \downarrow$ & $1.14 E-06$ \\
\hline Urine & 11.1 & 162.0532 & $\mathrm{C}_{9} \mathrm{H}_{8} \mathrm{NO}_{2}^{+}$ & Indole-3-carboxylic acid ${ }^{b}$ & $144.0414,116.0463,89.0382$ & $1.62 \downarrow$ & $2.00 E-09$ \\
\hline Urine & 4.09 & 184.0590 & $\mathrm{C}_{8} \mathrm{H}_{10} \mathrm{NO}_{4}^{+}$ & 4-Pyridoxic acid $b$ & $166.0469,148.0400$ & $1.41 \downarrow$ & $4.19 E-14$ \\
\hline Urine & 6.91 & 185.1245 & $\mathrm{C}_{5} \mathrm{H}_{16} \mathrm{NO}_{4} \mathrm{P}^{+}$ & Phosphorylcholine ${ }^{b}$ & 126.0902 & $1.55 \downarrow$ & $7.13 E-08$ \\
\hline Urine & 7.97 & 188.0692 & $\mathrm{C}_{11} \mathrm{H}_{10} \mathrm{NO}_{2}^{+}$ & Indoleacrylic acid ${ }^{b}$ & 142.0651 & $1.05 \uparrow$ & $1.71 E-06$ \\
\hline Urine & 8.82 & 190.0482 & $\mathrm{C}_{10} \mathrm{H}_{8} \mathrm{NO}_{3}^{+}$ & Kynurenic acid ${ }^{a, b}$ & $\begin{array}{c}162.0527,144.0431 \\
116.0464,89.0357\end{array}$ & $1.86 \downarrow$ & $2.86 E-15$ \\
\hline Urine & 8.16 & 206.0430 & $\mathrm{C}_{10} \mathrm{H}_{8} \mathrm{NO}_{4}^{+}$ & Xanthurenic $\operatorname{acid}^{b}$ & $\begin{array}{c}178.0462,160.0367 \\
132.0415\end{array}$ & $2.22 \downarrow$ & $9.00 E-06$ \\
\hline
\end{tabular}

${ }^{a}$ Metabolites confirmed using standard compounds. ${ }^{b}$ Metabolites confirmed by literature or database searches and MS fragmentation. ${ }^{c} \mathrm{MS} / \mathrm{MS}$ fragments were obtained with a CE of $30 / 10 \mathrm{eV}$, respectively. ${ }^{d}$ VIP is variable importance in the projection obtained from OPLS-DA with a threshold of 1.0. ${ }^{e} p$ value of the independent $t$-test between the control and model group.

and hydrazine-treated groups are summarized in Figure 5(b). To achieve more discriminative power, a biomarker group was generated including 6 upregulated (creatine, tryptophan, $N$-acetylhistidine, L-carnitine, pyroglutamic acid, and indoleacrylic acid) and 10 downregulated (prolinebetaine, L-acetylcarnitine, pipecolic acid, xanthurenic acid, trigonelline, kynurenic acid, indole-3carboxylic acid, phosphorylcholine, 4-pyridoxic acid, and thymine) metabolites with AUC $>0.85$. These biomarkers were further analyzed by binary logistic regression, followed by ROC curve analysis, which provided an AUC of 1 . In addition, both specificity and sensitivity calculated at best cut-off points could reach 100\% (S5 Fig). These findings demonstrate that this group of combined biomarkers showed a more preferable discrimination capability between healthy and liver-injured rats.

3.7. Biological Significance of the Identified Potential Biomarkers. Tyrosine and tryptophan have been shown to be associated with thioacetamide-induced liver fibrosis [38]. In the tryptophan metabolism pathway, lower levels of kynurenic acid and xanthurenic acid have been confirmed to indicate disturbances in liver function and energy-related metabolism [39, 40]. Furthermore, downregulation of indoleacrylic acid and indole-3-carboxylic acid might result from a tryptophan metabolism disorder involved in liver diseases [41].

Elevated creatine kinase and transaminase levels have been found in statin-induced liver damage [42]. In the current study, a dramatic decrease of urinary pipecolic acid was observed in hydrazine-induced liver injury. In addition, the upregulation of plasma pipecolic acid has been reported in chronic liver disease patients almost paralleling the severity of liver damage [43]. Pyroglutamic acid, an intermediate of the endogenous tripeptide glutathione biosynthesis pathway, is well documented as a hepatotoxicity biomarker following acetaminophen exposure $[10,44]$.

Trigonelline is an effective lipid-lowering agent that occasionally causes hepatic failure [45]. Previous studies have demonstrated that trigonelline is decreased by acute and chronic acetaminophen administration [46]. 4-Pyridoxic acid, the catabolic product of vitamin B6, regulates transsulfuration reactions for glutathione production and aberrant vitamin B-dependent hepatic methionine metabolism [47]. Moreover, 4-pyridoxic acid, indoleacrylic acid, and tryptophan have been shown to have a great influence on oxidative stress as well as on liver and renal dysfunction induced by pesticides [48].

Compared with the healthy controls, the liver-injured rats demonstrated significantly higher serum levels of 


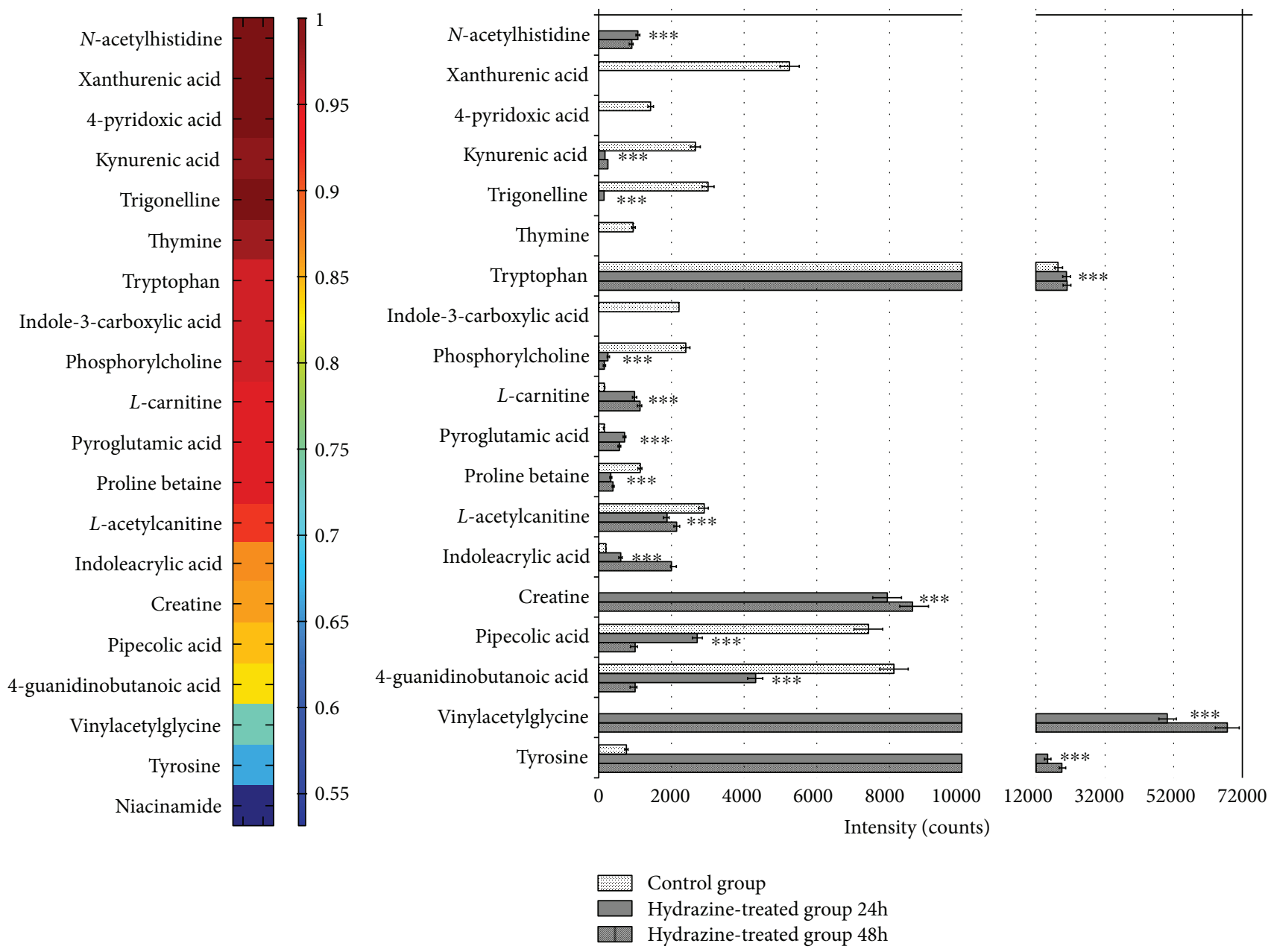

(a)

(b)

FIGURE 5: (a) Visualization of the discriminatory powers of individual potential biomarkers (AUC values $>0.8$ ). Heat map showing the discriminatory capacity of each metabolite estimated by the AUC. Colors correspond to AUC values; red and blue represent high and low values, respectively. (b) Concentration changes of potential biomarkers in the control and liver-injured groups $\left({ }^{* * *} p<0.001\right)$.

L-carnitine, while the level of acetylcarnitine decreased. Carnitine is reported to be essential for fatty acid metabolism and transport of activated long-chain fatty acids to $\beta$-oxidation sites in the mitochondria [49]. In rats, L-acetylcarnitine has been shown to increase the endogenous antioxidant defense mechanism and thus protect the animals from radiation-induced liver toxicity [50]. Therefore, elevated carnitine and decreased acylcarnitine levels might be important indicators for oxidation disturbances of long-chain fatty acids that are closely related to liver injury. Furthermore, increased levels of L-dihydroorotic acid and decreased levels of thymine have been demonstrated to result from a pyrimidine metabolism disorder in the development of liver diseases [51].

Elevated phosphorylcholine concentrations were observed in human hepatic tumor patients due to reconstitution of phospholipids in the injured membrane, and the opposite change was found in liver-injured rats [52]. These potential biomarkers indicate that perturbations of amino acids, glutathione metabolism, vitamins, fatty acids, pyrimidine, and lipid metabolism might be important in liver dysfunction.
Additionally, metabolomics pathway analyses confirmed that phenylalanine, tyrosine, and tryptophan biosynthesis as well as tyrosine metabolism had great interactions in hydrazineinduced liver injury in rats (Figure 6).

\section{Conclusions}

We investigated the application of an RRLC-MS/MS-based metabolomics method for hepatotoxicity evaluation in rats. To achieve a better discriminatory capability, a biomarker group was built by assembling biomarkers with high diagnostic value based on ROC analysis and a logistic regression model. The sensitivity and specificity of prediction were significantly improved by a group of biomarkers consisting of seven upregulated and ten downregulated metabolites. These discriminating metabolites might be useful in understanding the pathogenesis mechanisms of liver injury and provide good prospects for drug-induced liver injury diagnosis clinically.

This study confirmed the feasibility of using an LCMS-based urine metabolomics platform to characterize 


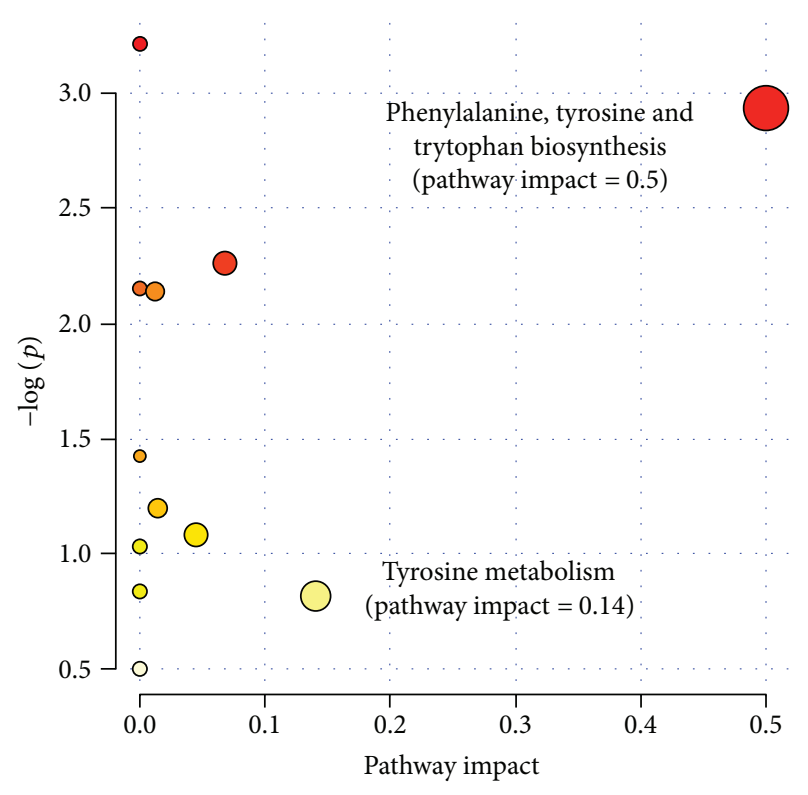

FIGURE 6: Metabolic pathway analysis for potential biomarkers related to liver-injured rats induced by hydrazine. The most relevant pathways are represented by large and dark nodes (pathway impact $>0.1$ ).

hydrazine-induced hepatotoxicity in rats. However, the specificity of these potential biomarkers must be further evaluated. Further studies will be applied to validate these biomarkers in larger cohorts of different patients.

\section{Conflicts of Interest}

The authors declare that they have no conflicts of interest.

\section{Funding}

This study was supported by grants (nos. 81100283 and 81341015) from the National Natural Science Foundation of China, a project (7142065) from the Beijing Municipal Natural Science Foundation and Beijing Municipal Administration of Hospitals' Youth Programme (QML20150305).

\section{Supplementary Materials}

S1 Table: the parameters of detailed preprocessing data applied in MZmine. S2 Table: method precisions (RSD) $(n=6)$ of LC-MS analysis for serum and urine samples. S3 Table: summary of potential biomarkers found between serum and urine samples of healthy rats and liver-injured rats by RRLC-ESIMS analysis in positive-ion mode. S1 Fig: overlaid extracted ion chromatograms of standard compounds in various test mixtures using RRLC-MS analysis during the whole sample batch $(n=6)$. Six standard compounds, (a) adenosine, (b) tanshinone IIA, (c) tramadol, (d) isosorbide mononitrate, (e) digitoxin, and (f) telmisartan, were selected to prepare various test mixtures for the (A) serum and (B) urine samples. They all provide an overall idea of the system stability during the run. S2 Fig: quality control (QC) plots of six randomly repeated runs of RRLC-(+)
ESIMS generated by principal component analysis for serum (A) and urine (B). Peak area deviation could be evaluated by the distribution of the runs. $x$-axis: run order; $y$-axis: standard deviation. (A1) and (B1) are QC plot of principal component analysis; (A2) and (B2) are QC plot for the first component. (A3) and (B3) are QC plot for the second component. S3 Fig: validation plots of the PLS-DA models obtained by using 100 permutation tests and T-predicted scatter plots of the OPLS-DA model for serum (A-B) and urine (C-D) samples. (The symbols are as follows: red circle $=\mathrm{C}$ healthy rats; brown circle $=\mathrm{M}$ liver-injured rats induced by hydrazine at $24 \mathrm{~h}$ postdosing; and blue circle $=$ FM liver-injured rats induced by hydrazine at $48 \mathrm{~h}$ postdosing.) S4 Fig: visualization of the discriminatory powers of potential biomarkers. (A) The eight upregulated metabolites provided AUC values of 0.667 to 1 . (B) The 11 downregulated metabolites provided AUC values of 0.838 to 1. S5 Fig: ROC curve of the combined potential biomarkers (AUC $>0.85$ ) that provided an AUC of 1 and sensitivities and specificities that reached $100 \%$, respectively. (Supplementary Materials)

\section{References}

[1] D. S. Goldberg, K. A. Forde, D. M. Carbonari et al., "Population-representative incidence of drug-induced acute liver failure based on an analysis of an integrated health care system," Gastroenterology, vol. 148, no. 7, pp. 13531361.e3, 2015.

[2] N. Chalasani, R. J. Fontana, H. L. Bonkovsky et al., "Causes, clinical features, and outcomes from a prospective study of drug-induced liver injury in the United States," Gastroenterology, vol. 135, no. 6, pp. 1924-1934.e4, 2008.

[3] R. Idilman, M. Bektas, K. Cinar et al., "The characteristics and clinical outcome of drug-induced liver injury: a single-center experience," Journal of Clinical Gastroenterology, vol. 44, no. 6, pp. e128-e132, 2010.

[4] M. Robles, E. Toscano, J. Cotta, M. Lucena, and R. J. Andrade, "Antibiotic-induced liver toxicity: mechanisms, clinical features and causality assessment," Current Drug Safety, vol. 5, no. 3, pp. 212-222, 2010.

[5] B. E. Senousy, S. I. Belal, and P. V. Draganov, "Hepatotoxic effects of therapies for tuberculosis," Nature Reviews Gastroenterology \& Hepatology, vol. 7, no. 10, pp. 543556, 2010.

[6] J. A. Agúndez, M. I. Lucena, C. Martínez et al., "Assessment of nonsteroidal anti-inflammatory drug-induced hepatotoxicity," Expert Opinion on Drug Metabolism \& Toxicology, vol. 7, no. 7, pp. 817-828, 2011.

[7] E. S. Björnsson, "Drug-induced liver injury due to antibiotics," Scandinavian Journal of Gastroenterology, vol. 52, no. 6-7, pp. 617-623, 2017.

[8] M. Chen, H. Bisgin, L. Tong et al., "Toward predictive models for drug-induced liver injury in humans: are we there yet?," Biomarkers in Medicine, vol. 8, no. 2, pp. 201-213, 2014.

[9] A. W. Nicholls, E. Holmes, J. C. Lindon et al., "Metabonomic investigations into hydrazine toxicity in the rat," Chemical Research in Toxicology, vol. 14, no. 8, pp. 975-987, 2001.

[10] S. Garrod, M. E. Bollard, A. W. Nicholls et al., "Integrated metabonomic analysis of the multiorgan effects of hydrazine 
toxicity in the rat," Chemical Research in Toxicology, vol. 18, no. 2, pp. 115-122, 2005.

[11] M. E. Bollard, H. C. Keun, O. Beckonert et al., "Comparative metabonomics of differential hydrazine toxicity in the rat and mouse," Toxicology and Applied Pharmacology, vol. 204, no. 2, pp. 135-151, 2005.

[12] D. J. Crockford, E. Holmes, J. C. Lindon et al., "Statistical heterospectroscopy, an approach to the integrated analysis of NMR and UPLC-MS data sets: application in metabonomic toxicology studies," Analytical Chemistry, vol. 78, no. 2, pp. 363-371, 2006.

[13] T. G. Klen $\varnothing$, B. Kiehr, D. Baunsgaard, and U. G. Sidelmann, "Combination of 'omics' data to investigate the mechanism(s) of hydrazine-induced hepatotoxicity in rats and to identify potential biomarkers," Biomarkers, vol. 9, no. 2, pp. 116-138, 2004.

[14] K. Bando, T. Kunimatsu, J. Sakai et al., "GC-MS-based metabolomics reveals mechanism of action for hydrazine induced hepatotoxicity in rats," Journal of Applied Toxicology, vol. 31, no. 6, pp. 524-535, 2011.

[15] M. Yu, Y. Zhu, Q. Cong, and C. Wu, "Metabonomics research progress on liver diseases," Canadian Journal of Gastroenterology and Hepatology, vol. 2017, Article ID 8467192, 10 pages, 2017.

[16] K. Kakisaka, Y. Yoshida, Y. Suzuki et al., "Serum markers for mitochondrial dysfunction and cell death are possible predictive indicators for drug-induced liver injury by direct acting antivirals," Hepatology Research, vol. 48, no. 1, pp. 78-86, 2017.

[17] J. K. Nicholson and I. D. Wilson, "Opinion: understanding 'global' systems biology: metabonomics and the continuum of metabolism," Nature Reviews Drug Discovery, vol. 2, no. 8, pp. 668-676, 2003.

[18] A. Sreekumar, L. M. Poisson, T. M. Rajendiran et al., "Metabolomic profiles delineate potential role for sarcosine in prostate cancer progression," Nature, vol. 457, no. 7231, pp. 910-914, 2009.

[19] D. Y. Chen, Y. M. Chen, H. J. Chien et al., "Metabolic disturbances in adult-onset still's disease evaluated using liquid chromatography/mass spectrometry-based metabolomic analysis," PLoS One, vol. 11, no. 12, article e0168147, 2016.

[20] M. S. Ahmad, M. Alsaleh, T. Kimhofer et al., "Metabolic phenotype of obesity in a Saudi population," Journal of Proteome Research, vol. 16, no. 2, pp. 635-644, 2017.

[21] L. Wang, S. Liu, W. Yang et al., "Plasma amino acid profile in patients with aortic dissection," Scientific Reports, vol. 7, article 40146, 2017.

[22] Z. An, Y. Chen, R. Zhang et al., "Integrated ionization approach for RRLC-MS/MS-based metabonomics: finding potential biomarkers for lung cancer," Journal of Proteome Research, vol. 9, no. 8, pp. 4071-4081, 2010.

[23] A. H. Emwas, C. Luchinat, P. Turano et al., "Standardizing the experimental conditions for using urine in NMR-based metabolomic studies with a particular focus on diagnostic studies: a review," Metabolomics, vol. 11, no. 4, pp. 872-894, 2015.

[24] A. Wuolikainen, P. Jonsson, M. Ahnlund et al., "Multiplatform mass spectrometry analysis of the CSF and plasma metabolomes of rigorously matched amyotrophic lateral sclerosis, Parkinson's disease and control subjects," Molecular BioSystems, vol. 12, no. 4, pp. 1287-1298, 2016.
[25] A. Vignoli, D. M. Rodio, A. Bellizzi et al., "NMR-based metabolomic approach to study urine samples of chronic inflammatory rheumatic disease patients," Analytical and Bioanalytical Chemistry, vol. 409, no. 5, pp. 1405-1413, 2017.

[26] I. D. Wilson, R. Plumb, J. Granger, H. Major, R. Williams, and E. M. Lenz, "HPLC-MS-based methods for the study of metabonomics," Journal of Chromatography B, vol. 817, no. 1, pp. 67-76, 2005.

[27] H. G. Gika, G. A. Theodoridis, J. E. Wingate, and I. D. Wilson, "Within-day reproducibility of an HPLC-MS-based method for metabonomic analysis: application to human urine," Journal of Proteome Research, vol. 6, no. 8, pp. 3291-3303, 2007.

[28] A. Scalbert, L. Brennan, O. Fiehn et al., "Mass-spectrometrybased metabolomics: limitations and recommendations for future progress with particular focus on nutrition research," Metabolomics, vol. 5, no. 4, pp. 435-458, 2009.

[29] M. S. Monteiro, M. Carvalho, M. L. Bastos, and P. Guedes de Pinho, "Metabolomics analysis for biomarker discovery: advances and challenges," Current Medicinal Chemistry, vol. 20, no. 2, pp. 257-271, 2013.

[30] M. Katajamaa and M. Oresic, "Processing methods for differential analysis of LC/MS profile data," BMC Bioinformatics, vol. 6, no. 1, p. 179, 2005.

[31] Y. Chen, J. Xu, R. Zhang et al., "Assessment of data preprocessing methods for LC-MS/MS-based metabolomics of uterine cervix cancer," The Analyst, vol. 138, no. 9, pp. 26692677, 2013.

[32] L. Eriksson, E. Johansson, N. Kettaneh-Wold, J. Trygg, C. Wikström, and S. Wold, Multivariate and Megavariate Data Analysis Part I: Basic Principles and Applications, Umetrics Academy, Sweden, 2001.

[33] Y. Chen, R. Zhang, Y. Song et al., "RRLC-MS/MS-based metabonomics combined with in-depth analysis of metabolic correlation network: finding potential biomarkers for breast cancer," The Analyst, vol. 134, no. 10, pp. 2003-2011, 2009.

[34] J. A. Swets, "Measuring the accuracy of diagnostic systems," Science, vol. 240, no. 4857, pp. 1285-1293, 1988.

[35] E. S. Lightcap and R. B. Silverman, "Slow-binding inhibition of $\gamma$-aminobutyric acid aminotransferase by hydrazine analogues," Journal of Medicinal Chemistry, vol. 39, no. 3, pp. 686-694, 1996.

[36] S. Wiklund, E. Johansson, L. Sjöström et al., "Visualization of GC/TOF-MS-based metabolomics data for identification of biochemically interesting compounds using OPLS class models," Analytical Chemistry, vol. 80, no. 1, pp. 115-122, 2008.

[37] L. Eriksson, E. Johansson, N. Kettaneh-Wold, J. Trygg, C. Wikström, and S. Wold, Multivariate and Megavariate Data Analysis Part II: Advanced Applications and Method Extensions, Umetrics, Sweden, 2006.

[38] D. D. Wei, J. S. Wang, P. R. Wang, M. H. Li, M. H. Yang, and L. Y. Kong, "Toxic effects of chronic low-dose exposure of thioacetamide on rats based on NMR metabolic profiling," Journal of Pharmaceutical and Biomedical Analysis, vol. 98, pp. 334-338, 2014.

[39] L. Rios-Avila, H. F. Nijhout, M. C. Reed, H. S. Sitren, and J. F. Gregory 3rd, "A mathematical model of tryptophan metabolism via the kynurenine pathway provides insights into the effects of vitamin B-6 deficiency, tryptophan loading, and induction of tryptophan 2,3-dioxygenase on tryptophan metabolites," The Journal of Nutrition, vol. 143, no. 9, pp. 1509-1519, 2013. 
[40] X. Sun, W. Xu, Y. Zeng et al., "Metabonomics evaluation of urine from rats administered with phorate under longterm and low-level exposure by ultra-performance liquid chromatography-mass spectrometry," Journal of Applied Toxicology, vol. 34, no. 2, pp. 176-183, 2014.

[41] F. Hartmann, E. Freiberg, and W. Ruge, "Indolepatterns in the urine of liver patients," Klinische Wochenschrift, vol. 40, no. 14, pp. 721-729, 1962.

[42] L. Li, Y. Ma, X. B. Geng et al., "Drug-induced acute liver injury within 12 hours after fluvastatin therapy," American Journal of Therapeutics, vol. 23, no. 1, pp. e318-e320, 2016.

[43] H. Kawasaki, T. Hori, M. Nakajima, and K. Takeshita, "Plasma levels of pipecolic acid in patients with chronic liver disease," Hepatology, vol. 8, no. 2, pp. 286-289, 1988.

[44] S. Geenen, C. Guallar-Hoyas, F. Michopoulos et al., "HPLCMS/MS methods for the quantitative analysis of 5-oxoproline (pyroglutamate) in rat plasma and hepatic cell line culture medium," Journal of Pharmaceutical and Biomedical Analysis, vol. 56, no. 3, pp. 655-663, 2011.

[45] E. H. Ali, B. McJunkin, S. Jubelirer, and W. Hood, "Niacin induced coagulopathy as a manifestation of occult liver injury," The West Virginia Medical Journal, vol. 109, no. 1, pp. 12-14, 2013.

[46] J. Sun, L. K. Schnackenberg, R. D. Holland et al., "Metabonomics evaluation of urine from rats given acute and chronic doses of acetaminophen using NMR and UPLC/MS," Journal of Chromatography B, vol. 871, no. 2, pp. 328-340, 2008.

[47] C. H. Halsted, "B-vitamin dependent methionine metabolism and alcoholic liver disease," Clinical Chemistry and Laboratory Medicine, vol. 51, no. 3, pp. 457-465, 2013.

[48] L. Du, S. Li, L. Qi et al., "Metabonomic analysis of the joint toxic action of long-term low-level exposure to a mixture of four organophosphate pesticides in rat plasma," Molecular BioSystems, vol. 10, no. 5, pp. 1153-1161, 2014.

[49] A. Kępka, S. D. Szajda, N. Waszkiewicz et al., "Carnitine: function, metabolism and value in hepatic failure during chronic alcohol intoxication," Postępy Higieny i Medycyny Doświadczalnej, vol. 65, pp. 645-653, 2011.

[50] H. H. Mansour, "Protective role of carnitine ester against radiation-induced oxidative stress in rats," Pharmacological Research, vol. 54, no. 3, pp. 165-171, 2006.

[51] H. Sun, A. H. Zhang, D. X. Zou, W. J. Sun, W. XH, and X. J. Wang, "Metabolomics coupled with pattern recognition and pathway analysis on potential biomarkers in liver injury and hepatoprotective effects of yinchenhao," Applied Biochemistry and Biotechnology, vol. 173, no. 4, pp. 857-869, 2014.

[52] T. Ogiso, T. Kobayashi, K. Kuhara, and Y. Kato, "Effect of "drugs for liver disease" on hepatotoxic action of carbon tetrachloride II. Effect of protoporphyrin and phosphorylcholine on microsomal drug-metabolizing enzyme activities and the components in injuried liver," The Japanese Journal of Pharmacology, vol. 25, no. 4, pp. 411-421, 1975. 


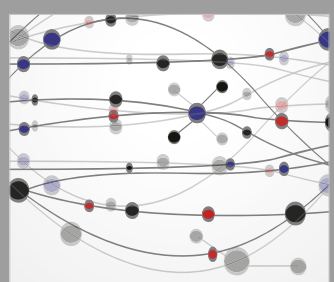

The Scientific World Journal
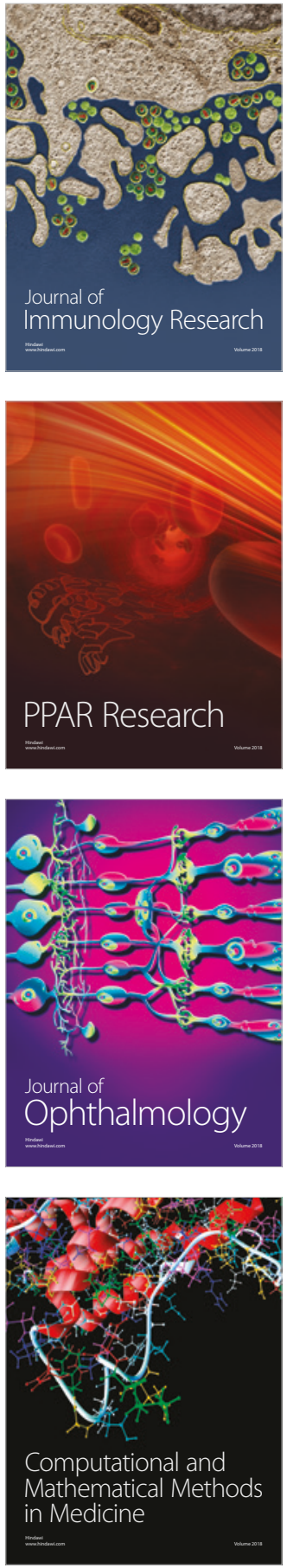

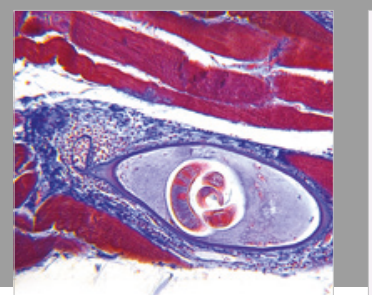

Gastroenterology Research and Practice

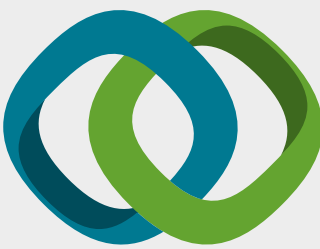

\section{Hindawi}

Submit your manuscripts at

www.hindawi.com
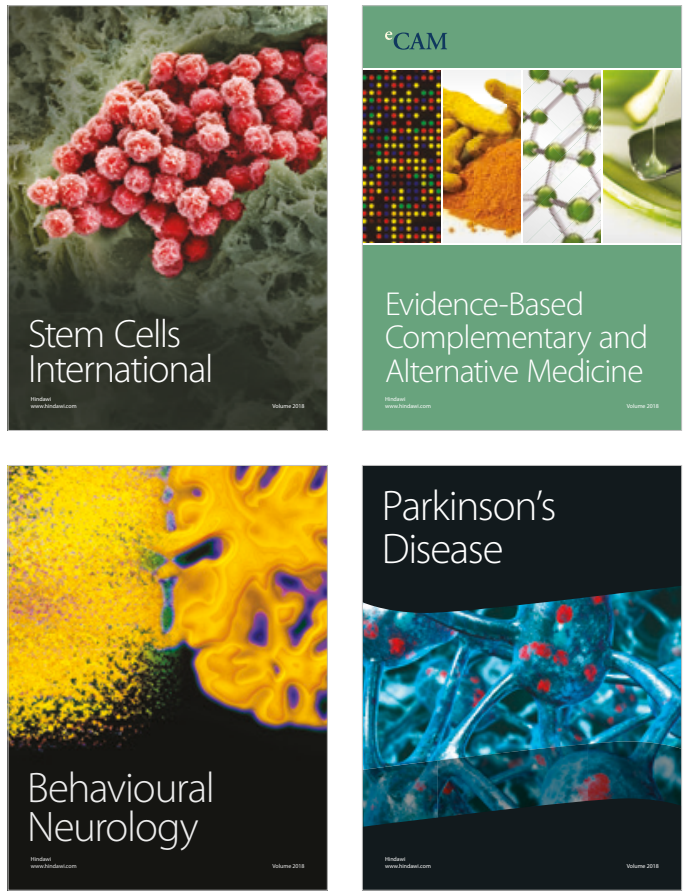

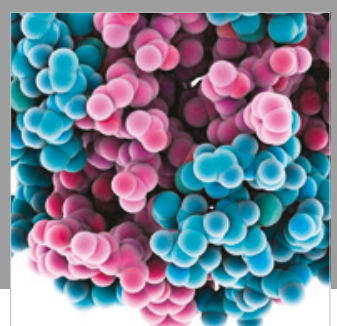

ournal of

Diabetes Research

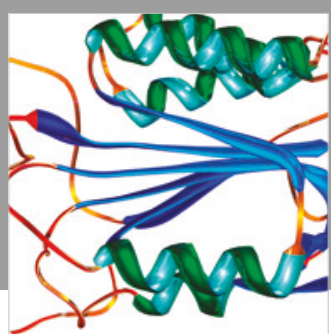

Disease Markers
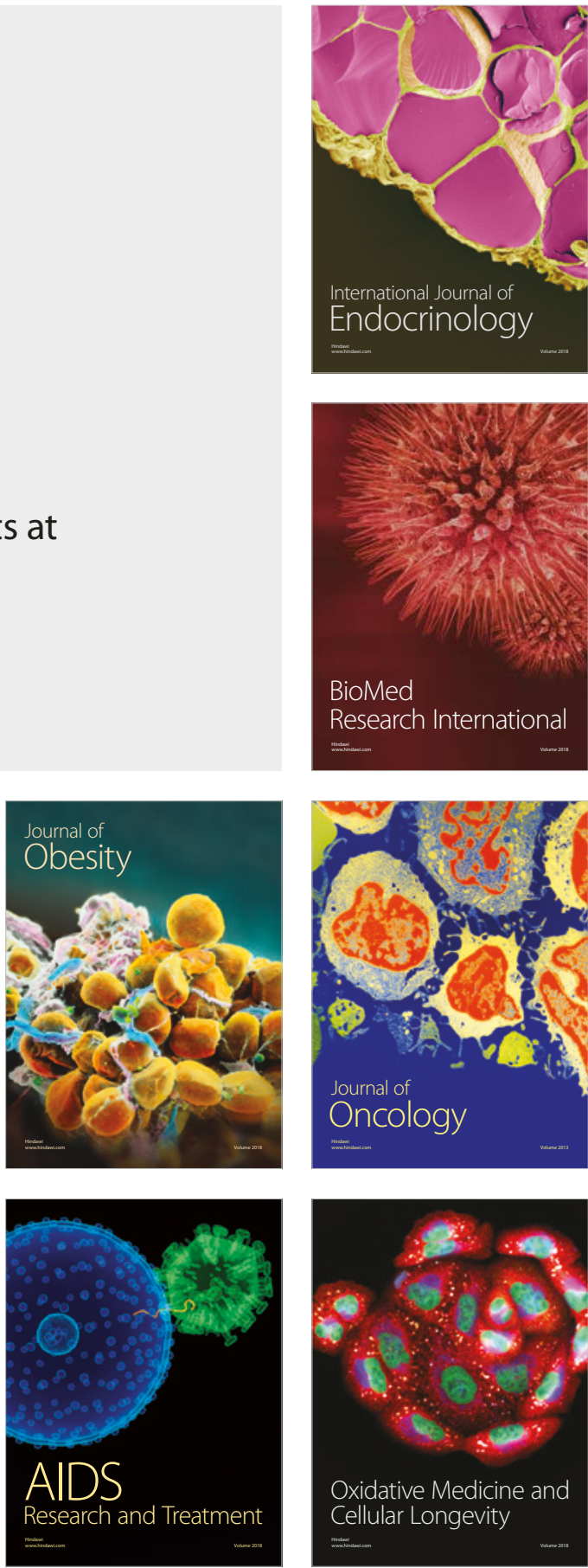